\title{
An Examination of Chemical Composition and Molecular Properties of Grape Berry Skin Flavonoids
}

\author{
Vladimir Sadovoy $^{1^{*}}$, Arcadiy Silantyev ${ }^{2}$ Magomed Selimov $^{3}$, Tatiana Shchedrina $^{4}$ \\ ${ }^{1}$ Stavropol Technological Institute of Service, Stavropol, Russia; ${ }^{2}$ Povolzhskiy State University of Telecommunications and Infor- \\ matics in Stavropol, Povolzhskiy, Russia; ${ }^{3}$ North Caucasus State Technical University, Stavropol, Russia; ${ }^{4}$ Pyatigorsk State Techno- \\ logical University, Pyatigorsk, Russia. \\ Email: *vsadovoy@yandex.ru
}

Received September $6^{\text {th }}, 2011$; revised October $6^{\text {th }}, 2011$; accepted October $14^{\text {th }}, 2011$.

\begin{abstract}
Using the molecular mechanics and semi-empirical methods, this study analyzes the main characteristics of the grape skin flavonoids: resveratrol, quercetin, rutin, catechin, epicatechin gallate, and epicatechin. We have carried out a comparative analysis of the molecular properties of the flavonoids and confirmed their antioxidant properties.
\end{abstract}

Keywords: Antioxidants, Molecular Properties, Grape Berry Skin

\section{Introduction}

In the modern concepts of nutrition, attention is being increasingly focused on minor components of food which largely determine its preventive and curative action. A current particular interest concerns the development of technologies for processing red grape pomace. One of the most representative groups of red-grape micronutrients is a large number of bio-antioxidants that reduce the activity of radical oxidation processes. In biological membranes, these processes mainly involve polyunsaturated fatty acids that are a forming part of phospholipids. At the initiation stage, free oxygen radicals cause the separation of the hydrogen atom in the alpha position in relation to the double bond. The presence of the double bond in the fatty acid weakens the $\mathrm{C}-\mathrm{H}$ bond in the adjacent carbon atoms, and thus facilitates the proton abstraction $(\mathrm{H} \bullet)$. The radical with the carbon in the center undergoes a molecular rearrangement, and further combines with oxygen to form peroxide radicals, capable of separating hydrogen from other fatty acids $[1,2]$.

The largest group among both natural and synthetic antioxidant compounds is comprised of the so-called phenolic antioxidants consisting of the aromatic ring associated with one or more hydroxyl groups. The presence of the aromatic ring causes the negative charge shift to the oxygen contained in the hydroxyl group. This shift facilitates an easier separation of hydrogen from $\mathrm{OH}$ groups and the formation of isomeric forms of phenoxy radical. Thus, phenolic antioxidants "intercept" peroxy and alkoxy radicals $[2,3]$. In studying the chemical composition of the berry skins of grapes "Levokumskij" was found that it contains $2.4 \%$ flavonoids (or $5.2 \%$ of dry residue). In the berry skins there are the most powerful antioxidants-resveratrol, quercetin, rutin, catechin, epicatechin, epicatechin gallate. We propose here some results on the composition of polyphenols in the grape skin and their molecular properties in order to assess their antioxidant activity.

Stavropol Territory is a region where more than $13 \%$ of the land mass is utilized for vineyards producing approximately $15 \%$ of Russia's gross wine grape harvest, which ranks the region third in the entire Russian Federation. The region's winemaking industry is represented by more than 30 manufacturers. One of the most widely cultivated technical grades of grapes in Stavropol Territory is "Levokumskij", with the area of vineyards producing this crop covering 1326.33 hectares (19.2\%) (second only to "Rkatsiteli" - a crop grown on 1521.27 hectares of land, or $22 \%$ of the region's vineyard area) [1].

\section{Materials and Methods}

\subsection{Materials}

This study examined the berry skin of red grapes "Levo- 
kumskij”, which grows in the North Caucasus region, Central region of Russia and the Far East. The chemical composition analysis was carried out using the skins of grapes "Levokumskij" grown in 2010 by ZAO "Zarja" (SJSC "Dawn") of Levokumskij district, Stavropol Territory.

\subsection{Methods}

Mass fraction of protein was determined using the Kjeldahl method; mass fraction of ashes was found by burning the trial sample and subsequent calcination of the ash content; to determine the mass fraction of moisture we used the Ohaus Moisture Analyzer MB45; determination of sugars was based on GOST (Federal Standard) 8756.13-87; mass concentration of titratable acids was determined by titration of the solution with sodium hydroxide; crude fiber content in grape pomace was calculated using the method of Henneberg and Shtoman; the quantitative determination of flavonoids in grape pomace extracts was performed through spectral analysis [4-7].

To examine the quantum chemical characteristics of flavonoids the molecular mechanics and semi-empirical methods in universal chemical packages ChemOffice Bio and HyperChem were applied.

\section{Results and Discussion}

Data analysis showed that the overall bagasse yield for the crop used for this study is $27.4 \%$. The bagasse consists of $25 \%$ grape seeds, $50 \%$ grape skins, and $25 \%$ grape stems (stalks). Whole red grapes and dry red wine (skin of whole grapes and the wine itself) contain the most powerful antioxidants - resveratrol, quercetin, rutin, catechin, epicatechin, epicatechin gallate, and others. Knowing the average level of crop yield for "Levokumskij" grapes (18.4 t/ha) [7], we can easily calculate how much of the flavonoids-rich grape skin is not used in food production, which just for Stavropol Territory alone comes out to an average of 3343.4 tons per year. The chemical composition of grape skins "Levokumskij" is shown in Table 1.

Studies indicate that the skin of grapes "Levokumskij" is a rich source of protein (12.7), fat (9.0) and flavonoids (5.2\% of dry residue). Active acidity $(\mathrm{pH})$ of grape marc equaled to 3.86 . It should be noted that the chemical composition of grape pomace (as well as grapes) varies depending on the type of culture, climate and agricultural practices of growing, technical equipment used for the grape processing, geographic area, soil, etc. However, the qualitative chemical composition of the bagasse components remains unchanged; hence the possibility of developing a technology for using this feedstock in the production of flavonoid-rich food supplements.

Based on the above, methods of computational chem-
Table 1. The chemical composition of grape berry skin Class "Levokumskij" $q \leq \mathbf{0 . 0 5}$.

\begin{tabular}{ccc}
\hline & \multicolumn{2}{c}{ Number } \\
\cline { 2 - 3 } Показатели & $\%$ & $\begin{array}{c}\% \text { for the dry } \\
\text { residue }\end{array}$ \\
Frotein & 5.8 & 12.7 \\
Cellulose & 4.1 & 9.0 \\
Ashes & 13.3 & 29.0 \\
Water & 4.1 & 9.0 \\
Mass fraction of sucrose & 54.2 & - \\
Mass concentration of titratable acids \\
(in terms of acetic acid)
\end{tabular}

istry were applied to examine the molecular properties of the following flavonoids: resveratrol, quercetin, rutin, catechin, epicatechin gallate, and epicatechin.

The examination of structure and molecular properties of resveratrol (Figure 1(b)) revealed lower charge values in the region of 7,8 and 17 oxygen atoms $(-0.239$, $-0.221,-0.231 \mathrm{eV}$, respectively), which confirms the possibility of using this chemical compound as a proton donor.

Distribution of surface charge density mapping revealed the overall hydrophobic properties of the molecule (Figure 1(b)), with the insignificant presence of hydrophilic areas, as evidenced by the resulting charge density value, equal to $0.05 \mathrm{eV}$. Similar results were obtained in studies of other flavonoids: quercetin, rutin, catechin, epicatechin and epicatechin gallate. It is evidenced by the total charge density value (see Table 2), which is close to zero.

In all cases, the antioxidant properties of these compounds were confirmed and it was established that hydrophobic areas are prevalent on the surface of charge density distribution.

Further, the molecular orbitals of resveratrol were examined. Figure 2 shows the results of the analysis of the highest occupied molecular orbital $(\mathrm{HOMO}=0)$.

These results indicate that the ionization energy of a resveratrol for $\mathrm{HOMO}=0$ has a low value and is equal to $8.807049 \mathrm{eV}$ (Figure 2(a)). This confirms the possibility of proton abstraction $(\mathrm{H} \bullet)$ from the oxygen atoms. Study of the surface configuration of the top-most occupied orbital (Figure 2(b)) showed that the transition of an electron in all three oxygen atoms $(7,8$ and 17$)$ to the lower unoccupied orbital (LUMO) requires spending of minimal activation energy, i.e. $\mathrm{HOMO}=0$ is bound with 


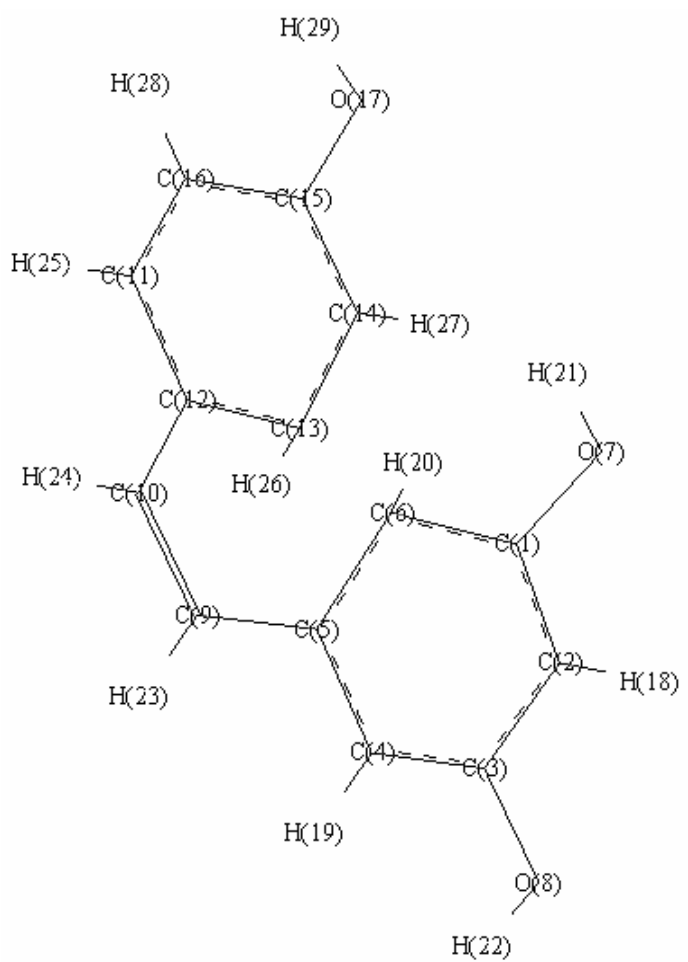

(a)

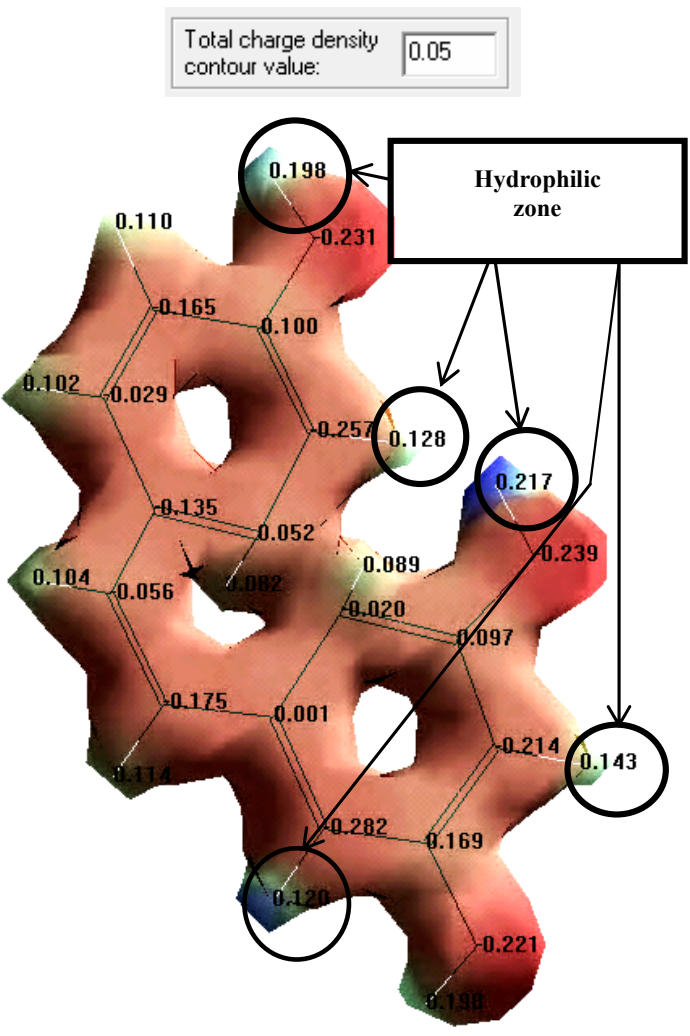

(b)

Figure 1. Study of surface charge density distribution of the molecule of resveratrol (a) structural formula; (b) surface charge density distribution.

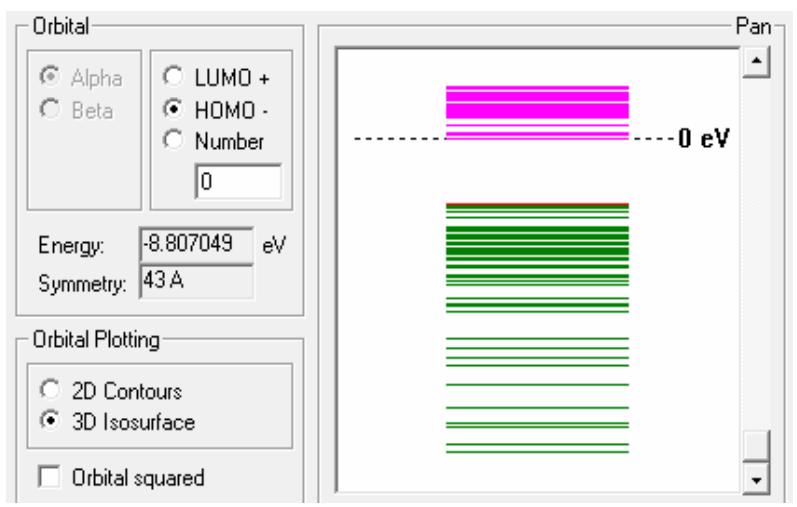

(a)

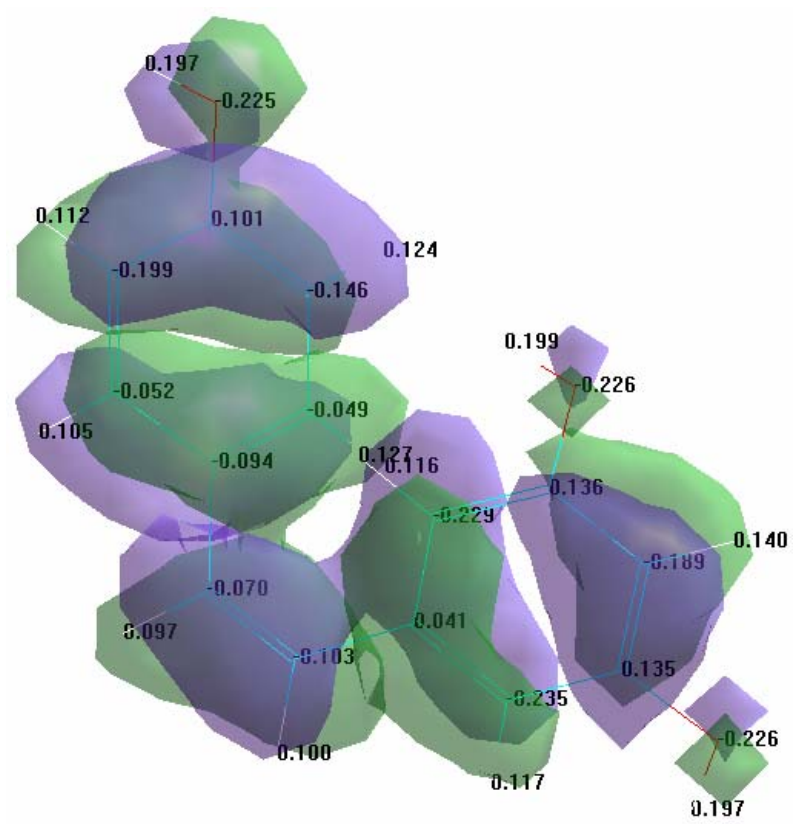

(b)

Figure 2. Molecular orbital study of resveratrol (a) analysis of energy performance; (b) the configuration of the highest occupied molecular orbital.

7, 8 and 17 atoms. It should be noted that the analysis of the HOMO for quercetin, rutin, catechin, epicatechin gallate, and epicatechin showed less pronounced antioxidant properties of flavonoids (Figures 3-4). An analysis of the configuration of HOMO showed that the electrons 11, 18, 21 of catechin atoms, electron 17 of quercetin atom, 30, 31, 32 of epicatechin gallate atoms, and electrons 19, 20 and 21 of oxygen atoms of epicatechin hydroxyl groups are not located on the top-most occupied molecular orbital. This means that the proton abstraction requires a greater energy than the energy of ionization.

Methods of molecular mechanics were applied to study the potential energy (Total Energy, Free Energy), 

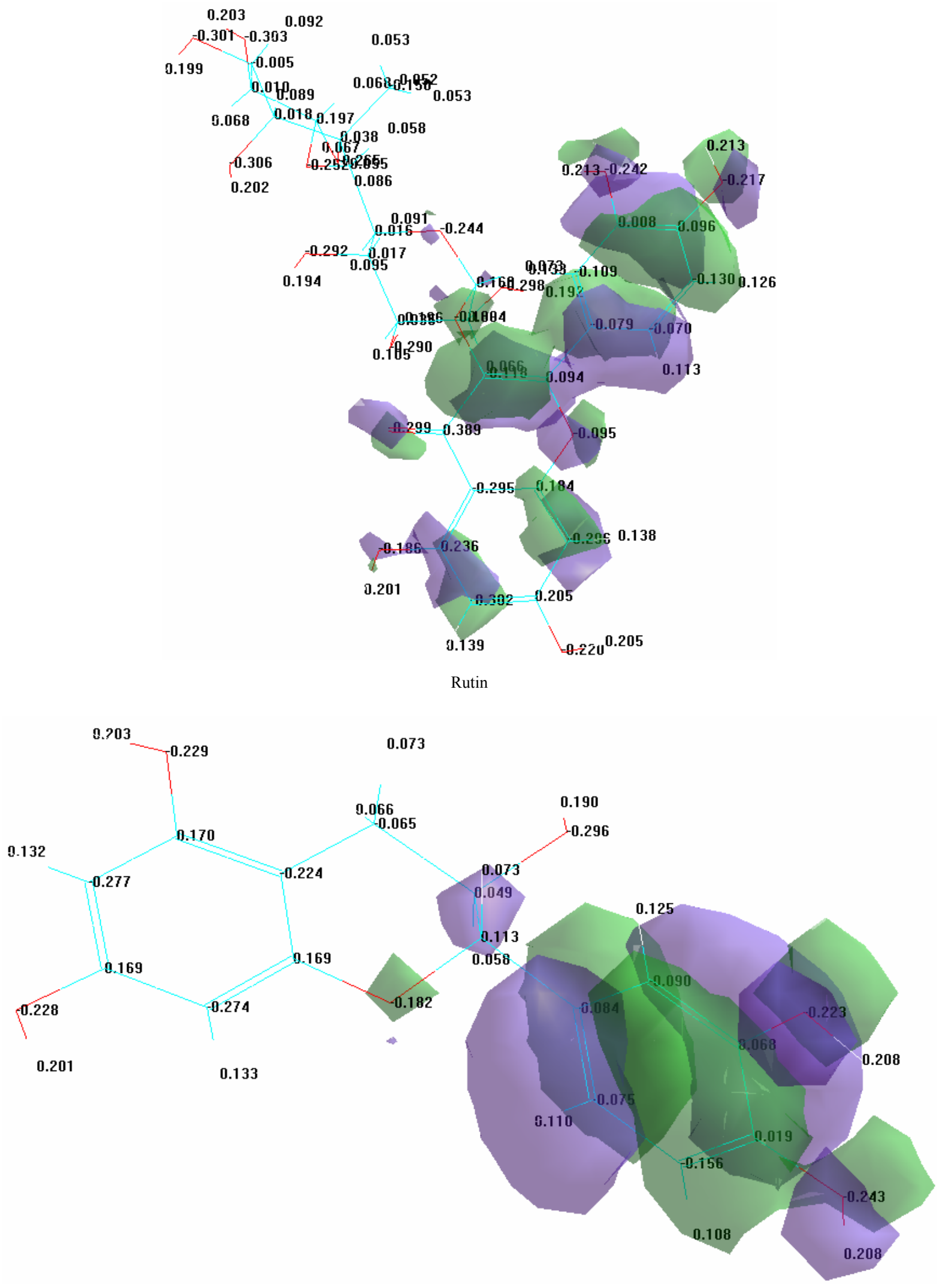

Catechin 


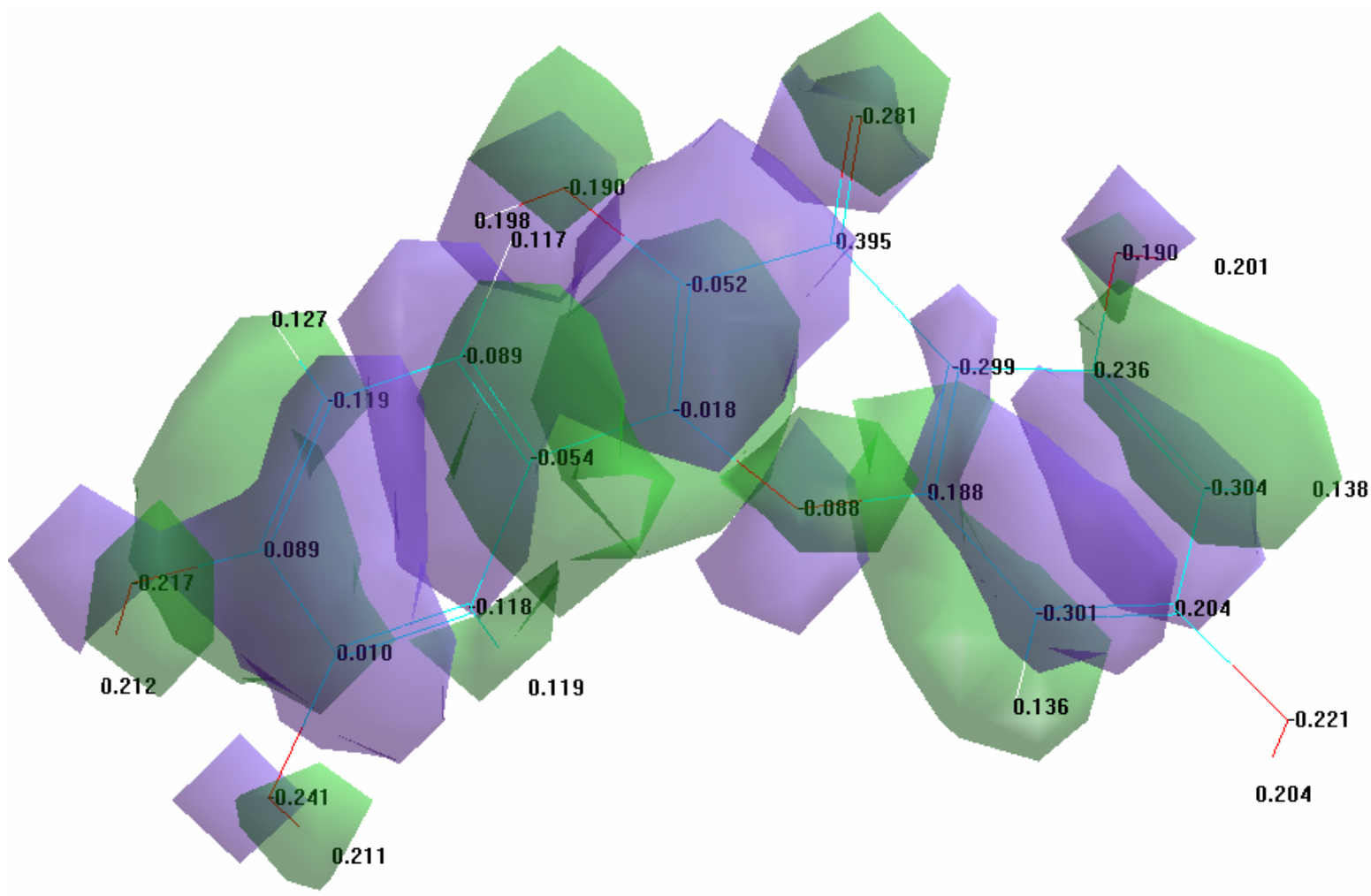

Quercetin

Figure 3. Top-most occupied molecular orbital of rutin, catechin and quercetin.

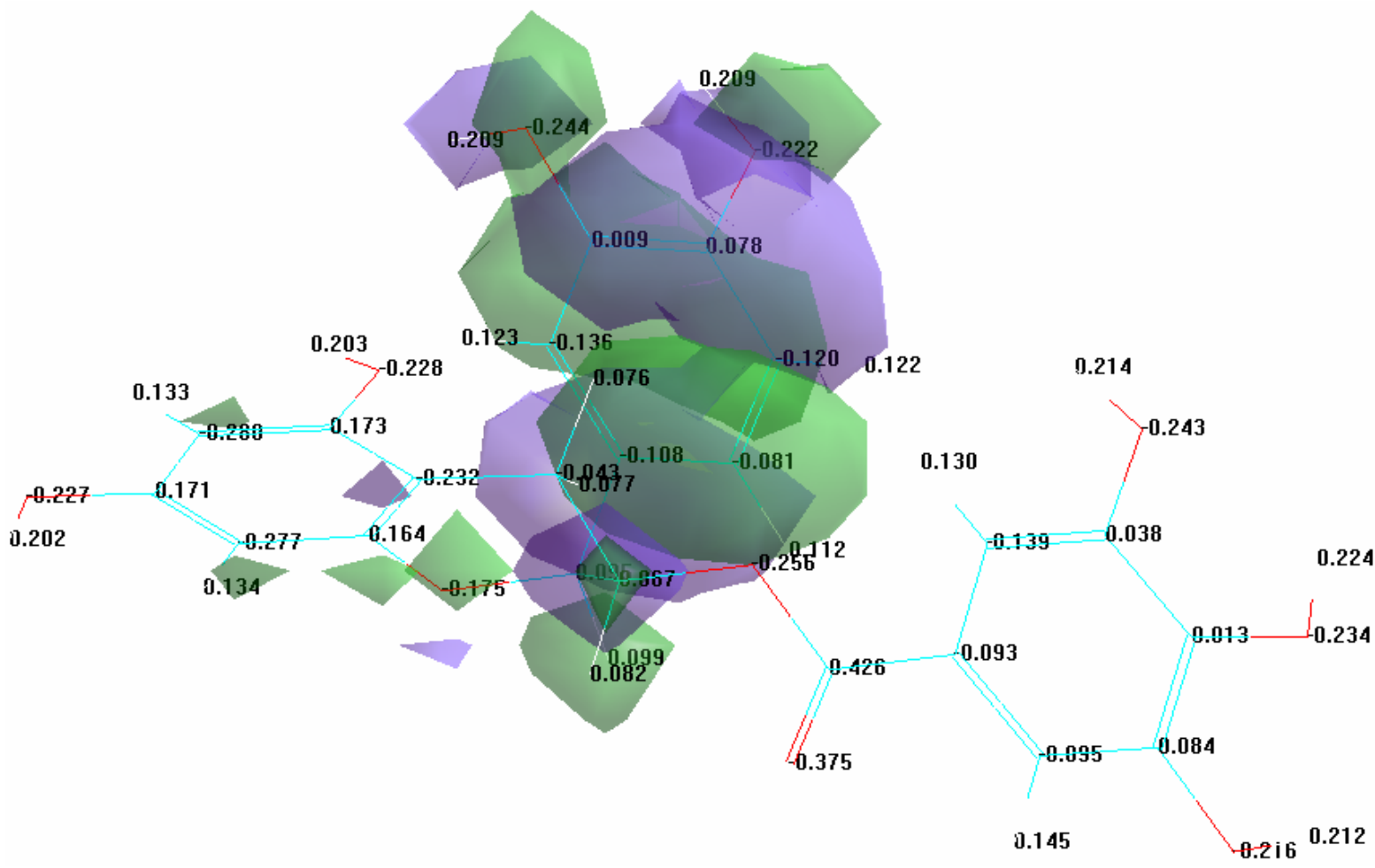

Epicatechingallate 


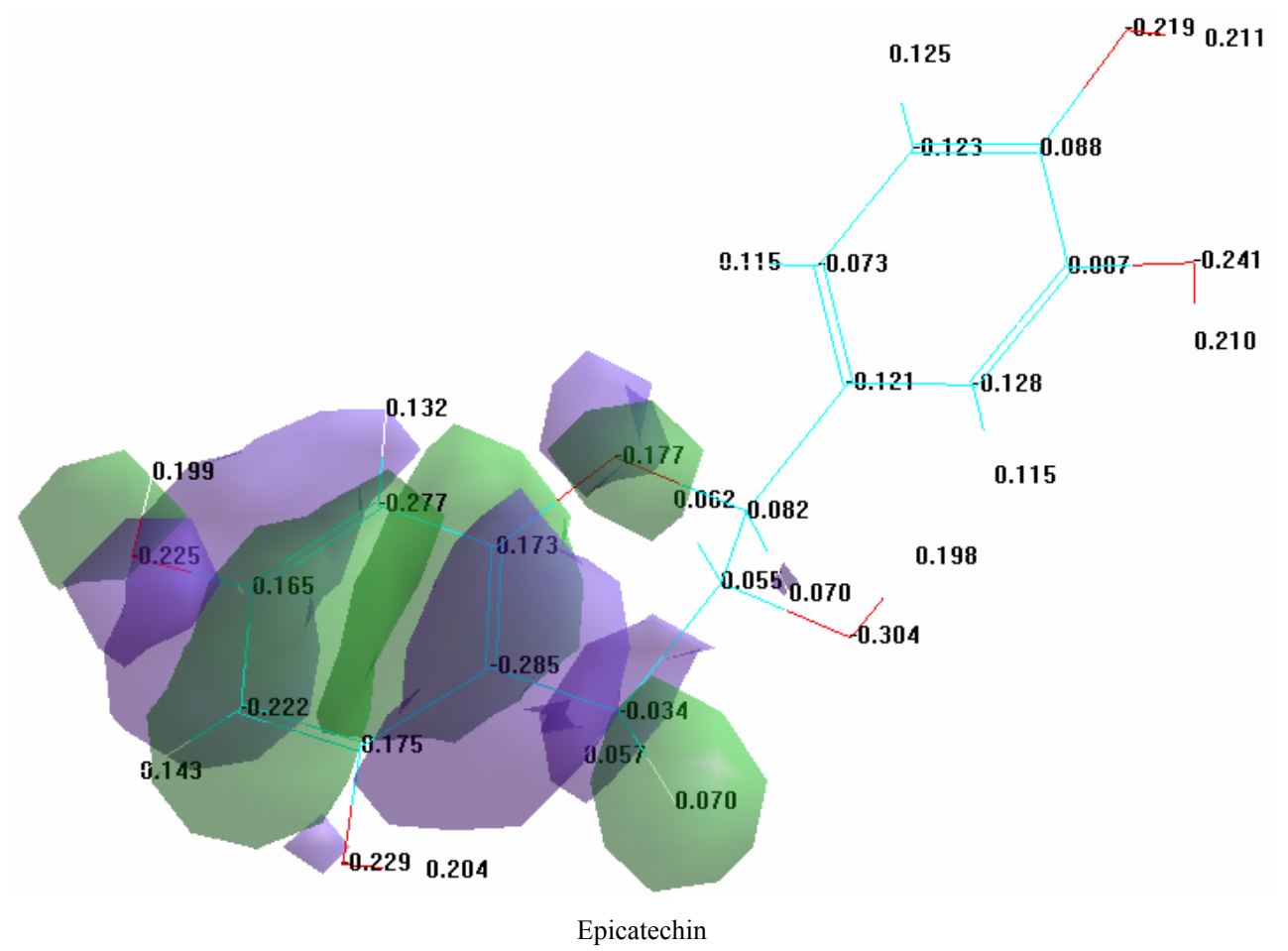

Figure 4. Top-most occupied molecular orbital of epicatechin gallate and epicatechin.

dipole moment and RMS Gradient of the flavonoid molecules in the grape bagasse under examination.

Figure 5 is a listing of the molecular properties of resveratrol.

The potential energy (Total Energy) value of the investigated model is quite small $(12.9081 \mathrm{kcal} / \mathrm{mol})$, and RMS gradient is close to zero $(0.04186 \mathrm{kcal} /(\AA \times \mathrm{mol})$, indicating the effective implementation of the procedure of minimizing the potential energy and showing that the system's energy properties are in a balanced state. Thevalue of the dipole moment (1.565 Debye) characterizes the uneven distribution of electron density (Figure 5). Similar calculations of molecular properties and energy characteristics of orbitals were performed for other grape skin flavonoids as well; with the results summarized in Table 2.

The data in Table 2 show that the most stable compound is a molecule of epicatechin, which has the lowest value of potential energy $(2.135 \mathrm{kcal} / \mathrm{mol})$; rutin is less stable $(43.610 \mathrm{kcal} / \mathrm{mol})$. All investigated molecules have a fairly uniform distribution of the charge of the electron density (dipole moment lies in the range of $1.495-4.321$ Debye units).

RMS gradient of all the molecules of flavonoids is close to zero $(0.042-0.098 \mathrm{kcal} /(\AA \times \mathrm{mol}))$, which indicates the effective optimization of the geometry of molecular structures. The final charge density is sufficiently low $(0.010-0.095 \mathrm{eV})$ to confirm the results of the ex-

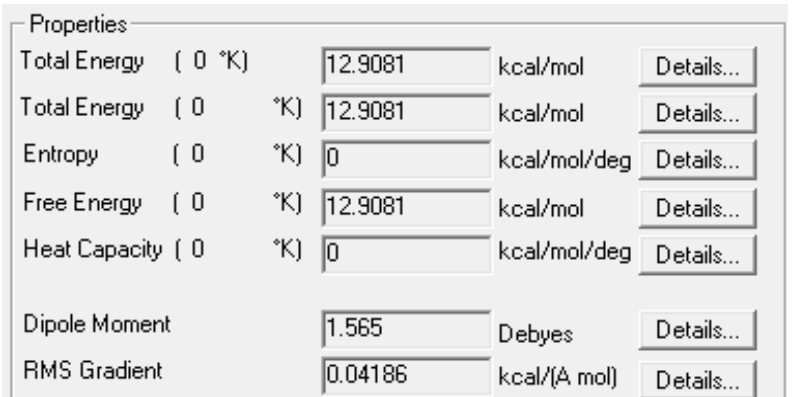

Figure 5. Basic molecular properties of resveratrol.

amination of charge density distribution surfaces, which suggests the prevalence of hydrophobic properties in the molecules under study. Therefore, the feasibility of extraction of flavonoids using polar solvents is minimal. The insignificant presence of hydrophilic areas suggests that the use of a mixture of polar and nonpolar solvents will ensure an efficient extraction of flavonoids.

The ionization energy has a low value (8.807 - 9.181 $\mathrm{eV}$ ), it is a type of binding energy, or, as it is sometimes called, the first ionization potential (I1), which is the lowest energy required to remove an electron from a free ground-state atom in its lowest energy (ground) state to infinity.

Thus, quantum chemical calculations carried out have confirmed that all tested flavonoids have antioxidant properties. Analysis of the charge density distribution 
Table 2. The main quantum chemical characteristics of the grape skin flavonoid molecules.

\begin{tabular}{lcccccc}
\hline \multicolumn{1}{c}{ Features } & \multicolumn{3}{c}{ Flavonoids } \\
\cline { 2 - 7 } & Resveratrol & Rutin & Catechin & Quercetin & $\begin{array}{c}\text { Epicatechin } \\
\text { gallate }\end{array}$ & Epicatechin \\
\hline Potential energy, kcal/mol & 12.908 & 43.610 & 2.014 & 20.370 & 5.521 & 2.135 \\
Dipole moment, Debye & 1.565 & 2.526 & 1.495 & 4.321 & 3.997 & 2.018 \\
RMS gradient, kcal $/(\AA \times \mathrm{mol})$ & 0.042 & 0.093 & 0.078 & 0.095 & 0.098 & 0.094 \\
Total charge density, eV & 0.050 & 0.095 & 0.010 & 0.010 & 0.010 & 0.010 \\
Ionization energy, eV & 8.807 & 9.181 & 8.856 & 8.906 & 8.879 & 9.040 \\
\hline
\end{tabular}

surface for the molecules showed the feasibility of using a mixture of polar and nonpolar solvents for the extraction of flavonoids from grape berry skins.

\section{REFERENCES}

[1] Sh. N. Guseynov, B. V. Chigrik, Z. S. Nagiev, N. G. Gordeyev and V. N. Gordeyev, "The All-Russia Scientific Research Institute of Wine Growing and Winemaking Named in Honour of J. I. Potapenko," Russian Academy of Agriculture, Novocherkassk, 2009.

[2] V. T. Kosyura, L. V. Donchenko and V. D. Nadykta, "The Essential Principles of Wine-Making," DeLiprint, Moscow, 2004. (in Russian)
[3] Zh. I. Abramova and G. I. Oksengendler, "Man and Antioxidants," Science, Leningrad, 1985. (in Russian)

[4] State Standards 8756.13-87, "The Fruit and Vegetable Processing Products: Methods of Sugars Determination.” (in Russian)

[5] State Standards 25555.0-82, "The Fruit and Vegetable Processing Products: Methods of Titrable Acidity Determination." (in Russian)

[6] State Standards P 52839-2007, "Feed Stocks. Methods of Raw Fiber Content Determination Using Intermediate Filtration." (in Russian)

[7] V. P. Georgievski, N. F. Komissarenko and S. E. Dmitruk, "Bioactive Components of Medicinal Plants," Science, Novosibirsk, 1990. (in Russian) 